\title{
Philosophiques
}

\section{Claude Gagnon : de la cabale des philosophes à la littérature des cabalistes}

\section{Bruno Roy}

Volume 7, numéro 1, avril 1980

URI : https://id.erudit.org/iderudit/203132ar

DOI : https://doi.org/10.7202/203132ar

Aller au sommaire du numéro

Éditeur(s)

Société de philosophie du Québec

ISSN

0316-2923 (imprimé)

1492-1391 (numérique)

Découvrir la revue

Citer cet article

Roy, B. (1980). Claude Gagnon : de la cabale des philosophes à la littérature des cabalistes. Philosophiques, 7(1), 77-84. https://doi.org/10.7202/203132ar d'utilisation que vous pouvez consulter en ligne.

https://apropos.erudit.org/fr/usagers/politique-dutilisation/ 


\title{
ÉTUDES CRITIQUES
}

\author{
CLAUDE GAGNON : \\ DE LA CABALE DES PHILOSOPHES \\ À LA LITTÉRATURE DES CABALISTES
}

par Bruno Roy

Depuis quelques années, Claude Gagnon se consacre à une entreprise de dévoilement d'autant plus remarquable qu'elle vise à déshermétiser les sciences occultes elles-mêmes. Cette entreprise a graduellement évolué, avec une régularité exemplaire, depuis une recherche sur l'existence même de son objet, exclu de tous les savoirs officiels, jusqu'à un regard porté sur le livre qui résume en lui toute la charge d'occultisme des traités apocryphes et pseudépigraphes suscités par la tradition alchimique, le Livre des figures de Nicolas Flamel.

En 1974, une première publication de Gagnon était consacrée à rassembler les membres disjoints de la bibliographie alchimique, constamment repoussée à la périphérie des savoirs et des pratiques orthodoxes. La Recherche bibliographique sur l'alchimie médiévale occidentale 1 , prudemment coiffée d'une épigraphe faisant état des "difficultés quasi insurmontables " qui se présentent en ce domaine, n'en rassemble pas moins 648 titres répartis en 18 sections. Ce premier effort, que Gagnon qualifiait avec raison de palingénésie, devait l'amener, dans une étude sur Les alchimistes et les spéculateurs ${ }^{2}$, à s'interroger sur la double exclusion dont les alchimistes ont été l'objet dans l'histoire occidentale: une première au Moyen Âge, en tant que faussaires, une seconde sous la plume des historiens modernes de la philosophie, en tant que «moyenâgeux». L'année où paraissait cette étude, l'auteur terminait une thèse de doctorat où tous ces problèmes d'exclusion (et même

1. Dans La sience de la nature: thénries et pratiques (Cahiers d'études médiévales, 2), Montréal-Paris, Bellarmin-Vrin, 1974, p. 155, 199.

2. Dans Guy-H. Allard (éd.), Aspects de la marginalité au Moyen Age, Montréal, L'Aurore, 1975 , p. $147-155$ 
d' « inclusion-exclusion ») allaient être abordés dans un cadre plus ample. La thèse se propose de faire l'autopsie d'un cas précis de censure dans l'histoire de la philosophie médiévale, celui de Nicolas Flamel $^{3}$. Il s'agit du plus célèbre alchimiste médiéval, dont l'ouvrage capital de «philosophie naturelle»a été écarté aussi bien des histoires modernes de la philosophie que du discours philosophique médiéval. La méthode qu'applique Gagnon à l'étude de ce problème est adaptée de l'Archéologie du savoir de Michel Foucault (Paris, Gallimard, 1969). Au terme d'une longue analyse, l'ouvrage attribué à Flamel apparaît à la fois comme apocryphe et comme authentique, selon les formations discursives auxquelles il a donné lieu. Mais comme le faisait remarquer un des lecteurs de la thèse (Robert Nadeau), l'analyse de Gagnon s'encombre parfois de notions étrangères à la méthode d'exégèse adoptée, en particulier celles d'auteur et d'authenticité. Or c'est précisément de ce côté que les recherches de Gagnon ont évolué depuis ce temps : préparation de matériaux de travail solides en vue de ses travaux historiques à venir, et formulation d'une hypothèse convaincante sur l'auteur du Livre des figures.

La première de ces démarches a été de publier trois textes flaméliens, sous un titre-fleuve digne de la tradition hermétique : Description du "Livre des Figures Hiéroglyphiques " attribué à Nicolas Flamel, suivie d'une réimpression de l'édition originale et d'une reproduction de sept talismans du "Livre d'Abrabam", auxquels on a joint le "Testament " authentique dudit Flamel". Comme l'auteur s'en explique à la fin du livre (p. 188), la présentation de ces trois textes (p.9-71 et 127-146) ne coïncide qu'en partie avec sa thèse précédente. Mais les textes qu'il publie sont intéressants à plus d'un titre. En effet les éditions modernes du Livre des figures (R. Alleau, 1970 ; E.-C. Flamand, 1973) ne nous donnaient jusqu'ici que des textes modernisés ; la reproduction en fac-similé de l'édition princeps (Paris, Guillemot, 1612) constitue donc un précieux outil de travail pour les historiens de l'alchimie. Quant aux sept

3. Analyse archéologique du "Livre des figures biéroglyphiques" attribué à Nicolas Flamel (1330-1418), thèse de Ph.D. (sciences médiévales), Montréal, Institut d'études médiévales, $1975,381 \mathrm{p}$.

4. Édité à Montréal, L'Aurore, 1977, $189 \mathrm{p}$. 
talismans qui n'étaient que décrits dans le texte de 1612 du Livre des figures, ils sont reproduits en couleurs d'après le manuscrit Paris, Arsenal, 3047 (XVII $^{\mathrm{e}}$ s.), le plus ancien témoin qu'on en connaisse. Dans sa Postface à la reproduction du Livre des figures, Gagnon apporte des précisions importantes sur la nature de ce livre et sur sa date de composition. (p. 135-137). Ce livre serait un faux, composé à partir d'un groupe d'écrits alchimiques latins parus entre 1572 et 1610 sous le titre collectif de Artis auriferae quam chemiam vocant.

Peu après la parution de ce livre, Gagnon formulait une hypothèse qui est appelée à bousculer tout le paysage de la littérature alchimique, et qu'il n'y a aucun risque à qualifier de découverte sensationnelle. Cette découverte tient en quelques lignes, qui ont fait l'objet d'une communication à l'École pratique des hautes études en $1977^{5}$, et d'une notice de la revue Anagrom ${ }^{6}$. On sait que le Livre des figures du Pseudo-Flamel est apparu dans l'imprimerie en 1612 en compagnie de deux autres traités alchimiques, le tout supposément traduit en français par «Pierre Arnauld de la Chevallerie, sieur poitevin ». Or cet «Arnauld» ne serait autre, d'après Gagnon, que le cabaliste bien connu Béroalde de Verville, dissimulant son nom (qui était d'ailleurs lui-même un pseudonyme) sous une anagramme. Béroalde est surtout connu par le Moyen de parvenir ; mais un bibliographe du XVIII siècle a noté, sur son exemplaire de la Bibliothèque françoise de La Croix du Maine, le titre d'un ouvrage de Béroalde qui présente une cascade d'anagrammes, précisément en rapport avec Flamel : Les aventures d'Ali el Moselan [ = Nicolas Flamel] surnommé dans ses conquêtes Slomnal Calife [= Nicolas Flamel], traduit de l'arabe de Rabi el Ulloe de Deon [ = Béroalde de Verville].

Il est impossible de dégager ici toutes les conséquences qu'aura cette découverte sur l'histoire et l'interprétation des textes alchimiques. Gagnon s'y est d'ailleurs déjà attaqué, en mettant en chantier l'édition critique du Livre des figures. Reste

5. C. Gagnon, Identification de l'auteur du "Livre des figures biéroglypbiques " attribué à Nicolas Flamel, dans l'Annuaire 1976-77 de l'École pratique des bautes études, IVe section, Paris, Sorbonne, 1977, p. 569-70 (séminaire d'histoire des sciences dirigé par Guy Beaujouan).

6. C. Gagnon, Déinuterte de l'identité de lauteur réel du "Livre des figures biéroglyphiques",

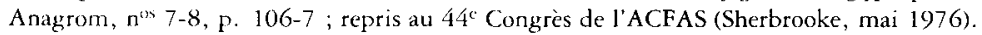


que déloger un livre de son temps littéraire n'est pas une mince affaire, a fortiori si le décalage est de deux siècles; on peut se demander par quelle vertu d'occultation les critiques précédant Gagnon n'ont pas su lire ce qui semble désormais une évidence. D'autres pistes d'évidence avaient d'ailleurs été soigneusement placées dans le texte par l'auteur du Livre des figures : des références explicites à des traités parus à la fin du $\mathrm{XVI}^{\mathrm{e}}$ siècle comme le Livre d'Hercule, certains textes de la Turba philosophorum et le De lapide philosophorum figurae et emblemata de Lambspringk (1599) ${ }^{7}$. Un texte est forcément postérieur aux textes qu'il cite; mais au lieu d'appliquer cette règle trop simpliste pour eux, les critiques s'affairaient à rechercher le manuscrit (grimoire) de Flamel, ou à attribuer sa perte à des causes mystérieuses, ou à prétexter que même si on le trouvait, on ne serait pas plus avancé puisque l'ouvrage n'était pas entré dans le domaine littéraire sous sa forme originale, mais sous celle d'une traduction. Dans cette sombre affaire, qui était la dupe? Sûrement pas Béroalde ni ses contemporains. Ni sans doute les hermétistes modernes, qui préferent laisser croire que l'alchimie, loin d'être née dans la paiennie musulmane, a pour ancêtre éponyme un excellent chrétien du XIV ${ }^{\mathrm{e}}$ siècle, au surplus théologien, philosophe et riche bourgeois. J'imagine plus aisément que certains se sont fort amusés à ce Livre des figures hiéroglyphiques : ce serait le cas de Béroalde, qui est à l'humour français ce que Rabelais est au comique; d'autres en ont profité, conformément à une tradition à qui il tarde de voir les "pierres " se transformer en pain; pour tous les autres, les lecteurs comme nous, c'est d'ignorance qu'il s'est agi. Une ignorance dans l'art immémorial de la lecture.

C'est que le cas Flamel n'est pas unique. Je pense à la plus célèbre encyclopédie du Moyen Âge, Sidrac ou la Fontaine de toutes sciences. S'il faut en croire son prologue, le texte français du Sidrac aurait été traduit à Tolède d'une version

7. Voir Gagnon, Description . .., p. 135-6. L'insistance que met le Pseudo-Flamel à se faire démasquer apparaît bien dans un passage comme celui-ci : "Moult plaist à Dieu procession/S'elle est faite en devotion : ce qui est quasi le commencement du Livre du Roy Hercules, traictant des couleurs de la pierre, intitulé L'iris, en ces termes : 'Operis processio multum Naturx placet, etc.' que jay mis là trut exprès pour les grands Cleris qui entendront liallusion » (Live des figures, éd. Gagnon, p. 87). 
latine faite d'après un texte "sarrasinois ", lui-même exécuté en Espagne sur une traduction latine d'un original grec. Scénario semblable dans le Secret des secrets, les Kiranides (traités sur les vertus des animaux, des piertes et des plantes), les Prophéties de Merlin, etc. Dans tous ces cas, la critique moderne s'est évertuée à rechercher des originaux inexistants, là où les auteurs ne voulaient signifier qu'une chose: qu'ils transmettaient une tradition. Et comme s'il ne suffisait pas à notre aveuglement d'avoir perdu le sens du topos de la traduction, nous n'avons pas non plus su lire les topoi du pèlerinage à Compostelle $^{8}$, du livre volé aux Juifs, et surtout celui du livre-objet.

Car il me faut dire comment est construit le Livre des figures, sous peine d'être moi-même un des accusés à ce procès en occultation intenté par Gagnon à tous les détenteurs de discours. Dans ce Livre donc, Flamel prend la parole et raconte comment, étant occupé à son métier d'écrivain public, il acheta d'occasion un vieux livre doré écrit sur des écorces, comportant trois cahiers de six folios, chaque cahier étant augmenté d'un septième folio enluminé. La couverture en était de cuivre, gravée de lettres étranges. Le texte du livre était en latin, et illustré de quatre autres miniatures: c'était le Livre d'Abraham. Flamel mit le livre en sureté et fit recopier les sept miniatures pour les faire voir aux grands clercs, lesquels n'y entendirent naturellement rien. Un certain maître Anseaume proposa une grille de lecture, mais tellement inadéquate que Flamel perdit son temps en "brouilleries » pendant 21 ans, à tenter de la rendre opératoire. L'exégèse véritable de ce livre devait lui être donnée par un Juif converti, lors d'un pèlerinage à Compostelle ; à la suite de quoi il réussit enfin à " accomplir aisément le magistère ». Pour fixer la mémoire de sa réussite, il fit construire au cimetière des Saints-Innocents un monument figuré, à l'imitation du livre doré du Juif Abraham». Toute cette histoire est racontée dans l'Avant-propos du Livre des figures; quant au corps du livre, c'est un commentaire des figures de cette "Arche»: commentaire théologique (chap. 1), puis philosophique (ch. 2-9).

8. Voir cependant, sur la ropique du pèlerinage, C. Gagnon, Analyse archéologique..., p. 187-8; Idem, Médecine, alibimie et pèlerinages, Critère, n 13 (1976), p. 157-172. 
Dans cette histoire, le lecteur apprend que Flamel a perdu autant d'années en expériences ratées qu'il y avait de folios au Livre d'Abrabam : il lui fallait annuler une à une les gloses erronées du maître parisien, pour devenir disponible à une révélation de type biblique (le juif converti). Mais que dire de ce Livre d'Abraham, matrice et modèle de tout le Livre des figures? Qui a jamais vu un livre d'écorces de $3 \times 7$ folios, à couverture de cuivre? La véritable alchimie est précisément ici : un livre est un train de rentrer à l'intérieur même de son étymologie (liber $=$ écorce) ; sa couverture est un minéral, et il contient des paroles. Au Moyen Âge, un axiome servait à préfacer les ouvrages éthico-scientifiques comme les lapidaires et les plantaires : "In petris, herbis vis est, sed maxima verbis. "Mais il y a ici beaucoup plus : une transmutation au terme de laquelle le végétal aura tiré à lui tous les règnes, absolument ( 3 fois 7 ). Le livre-écorce (liber), c'est donc le livre. Un livre unique et quasi divin, par sa parfaite cöncidence avec lui-même ; un livre si puissant qu'une simple imitation de lui, soit le texte principal du Livre des figures, suffira à accomplir une seconde transmutation. Et cette transmutation est la suivante: transformer un monument funéraire en document alchimique.

Il n'y a pas de doute que ce coup d'état littéraire a réussi. Dès le milieu du XVII ${ }^{e}$ siècle, on avait réussi à "retrouver" le Livre d'Abraham et à le publier . . . sur papier. La " poudre de projection » agissait aussi rétroactivement sur un document authentique de Nicolas Flamel, son testament. Qu'un tel coup de force ait été l'œuvre de Béroalde, il n'y a pas à s'en surprendre, car celui-ci est bien connu pour s'être essayé une autre fois à cet exercice de globalité, dans Le moyen de parvenir, cuure contenant la raison de tout ce qui a esté, est, et sera. Comme Béroalde le déclare lui-même au début ${ }^{9}$,

Je vous diray le secret des secrets : mais je vous prie afin qu'il soit secret, de vous embeguiner le museau du cadenac de taciturnité et escoutez, CE LIVRE EST LE CENTRE DE TOUS LES LIVRES : voilà la parole secrette, qui doit estre descouverte au temps d'Helie, artiste, ainsi que disent les Alquemistes.

9. Édition Paul Lacroix, Paris, 1889, I, c. 51. 
Et je serais porté à penser que dans le registre qui est celui de ce second livre, ce diable de Béroalde est «parvenu» à son but, quel que soit le sort que l'histoire littéraire moderne ait fait à ce chef-d'œuvre de l'humour français.

En fin de compte, le Flamel de Béroalde est beaucoup plus séduisant pour nous que le véritable bourgeois bigot, mégalomane et procédurier qui vivait au $\mathrm{XIV}^{\mathrm{e}}$ siècle. À chacun son Flamel : les lecteurs du XVII e siècle ont eu le leur, nous avons celui de Marguerite Yourcenar, qui ne le cède en rien au précédent, et les lecteurs de Spirou ont un Faiseur d'or pour les introduire aux merveilles du rêve alchimique.

Mais il ne s'ensuit pas que la philosophie métallique ait été inexistante au Moyen Âge. En attendant que paraisse son édition critique du Livre des figures, Claude Gagnon a préparé un rapport qui paraîtra bientôt sous le titre Alcbimie et philosophie au Moyen Âge. Perspectives et problemes ${ }^{10}$. Ce livre résulte de la mise en commun de deux rapports soumis en 1977 au Congrès de la Société Internationale pour l'Étude de la Philosophie Médiévale. La question posée était celle-ci : en quoi l'alchimie peut-elle contribuer à une meilleure compréhension de la philosophie médiévale? Les deux auteurs, Claude Gagnon et Chiara Crisciani, présentent une synthèse qui commence par situer l'alchimie dans les Histoires de la philosophie médiévale. La question proprement dite est ensuite abordée de deux manières : l'aspect littéraire des sciences dites occultes, et l'aspect doctrinal, lui-même divisé en deux parties: a) description de certains sélecteurs linguistiques permettant de situer épistémologiquement l'alchimie dans le savoir médiéval, b) examen du cas de Petrus Bonus de Ferrare, un médecin du XIVe siècle sympathique à l'alchimie. La dernière section de l'ouvrage tente de formuler la spécificité du projet alchimique au-delà de la diversité de l'aspect littéraire. Cet ouvrage est précieux non seulement pour sa très grande richesse bibliographique, mais aussi pour les prises de position des auteurs sur plusieurs sujets importants ${ }^{11}$. On ne peut

10. Édité à Montréal, L'Aurore, sous presse.

11. La question épistémologique, qui se sirue au centre des préoccupations de C. Gagnon, a fait l'objet d'une brève étude : La questron de l'épistémologie des alchimies médiévales: l'exemple de la rose et du rosaire, dans G. Mermier et E. DuBruck, Fifteenth-Century Studies, II, Ann Arbor, 1979, p. $91-94$. 
mieux le présenter et le recommander qu'en reprenant les termes de Guy Beaujouan, qui en a écrit la préface: "Le travail de C. Gagnon et de C. Crisciani ne livre pas à notre paresse des résultats définitivement acquis; il nous propose plutôt un art de lire, en philosophes, la littérature alchimique. »

Les grands succès de commerce que connaissent ces années-ci les ouvrages hermétiques ont de quoi nous inquiéter, compte tenu du petit nombre de ceux qui s'adonnent à des philosophies plus ouvertes. Mais Claude Gagnon, qui a établi ses recherches au point précis de rupture entre le philosophal et le philosophique, est bien placé pour instaurer bientôt une sorte de coincidentia oppositorum dont la fécondité sera sûrement très grande.

Institut d'études médiévales 